\title{
A Study on the Detection of Asynchronous State of the Synchronous Generator
}

\author{
Hyung-Joo Choi *, and Heung-Ho Lee **
}

\begin{abstract}
This paper includes new protection concepts and practices to avoid mechanical damage of three-phase transformer by asynchronous operation of synchronous generator. This failure is often caused just after synchronous generator was connected to the grid because of a malfunction of the controller or misconnections of the synchronous devices. The results of the studies on the analyzing the phenomenon of asynchronous operation experienced in Korea and rapidly detecting asynchronous state are described.
\end{abstract}

Keywords: Asynchronous detection, Surge admittance, Out-of-step protection, Generator protection

\section{Introduction}

\subsection{Synchronizing System}

The purpose of the synchronizing system is to match the voltages and phases between a synchronous generator and grid system sides. The magnitude of the voltage and deviation of its phase of the synchronous generator will be controlled respectively by the AVR (Automatic Voltage Regulator) and the GOV (Governor) in the synchronizing controller as shown in Fig. 1 [1].

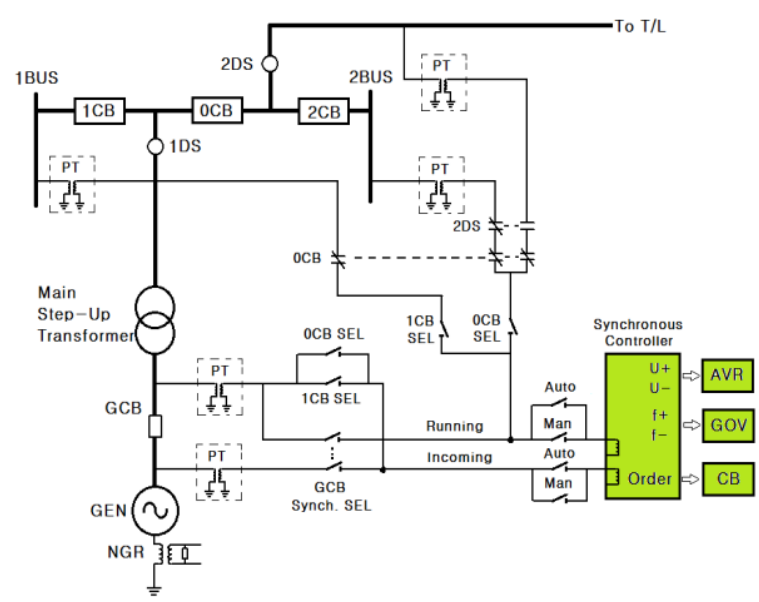

Fig. 1. Schematic of the Synchronizing Control System

* Dept. of electrical Eng. Korea Midland Power Company(KOMIPO), Korea (nimble@komipo.co.kr)

** Department of Electrical Engineering, Chung-Nam National University, Korea (leehh@cnu.ac.kr)

Received 14 October 2013; Accepted 2 November 2013

\subsection{Impact of asynchronous operation}

Large phase displacement and frequency differences result in generator hunting, a condition where the synchronizing torque accelerates the rotating system, overpowering the rotor damping, etc., resulting in the rotor swinging past the synchronous position under its own inertia. To produce the fluctuating torque required, the generator has to alternately supply and absorb sizeable synchronizing power with an initial transient which can involve currents in excess of generator rating [2].

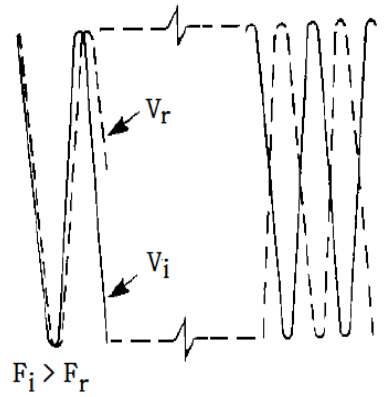

$$
\begin{aligned}
\mathrm{V}_{\mathrm{i}} & =\text { INCOMING VOLTAGE } \\
\mathrm{V}_{\mathrm{r}} & =\text { RUNNING VOLTAGE } \\
\mathrm{F}_{\mathrm{i}} & =\text { FREQUENCY OF } \mathrm{V}_{\mathrm{i}} \\
\mathrm{F}_{\mathrm{r}} & =\text { FREQUENCY OF } \mathrm{V}_{\mathrm{r}}
\end{aligned}
$$

Fig. 2. Phase error between incoming and running voltage

A serious damage of the three-phase main step-up transformer winding deformation has experienced several times in Korea. The main cause of damage was confirmed in malfunction of the synchronizing devices. Severe transient inrush fault currents are recorded $68.9 \mathrm{kA}$ in the fault recorder just after the synchronous generator was connected to the network under out of synchronism as shown in the following figure. The generator circuit breaker was opened at $455.6 \mathrm{~ms}$ by the loss of excitation protection. 


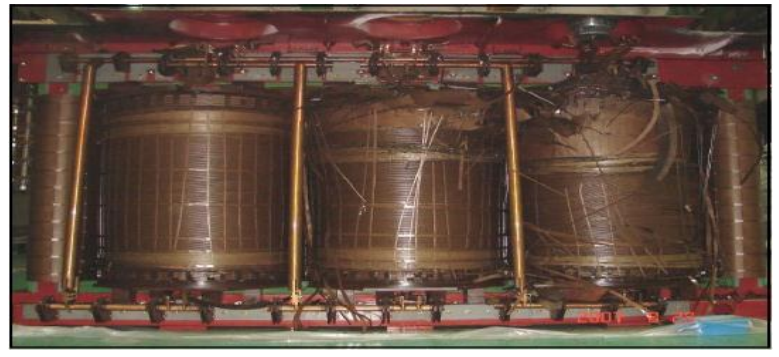

Fig. 3. Damaged three phase step-up transformer

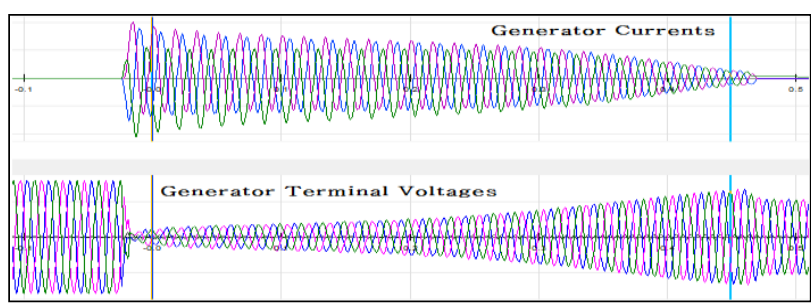

(a) Fault current and voltage of the synchronous generator

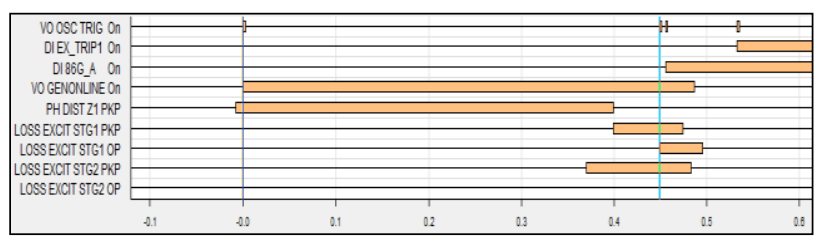

(b) Events of the digital signals

Fig. 4. Analog and Digital events of the Fault Recorder

The first peak of an inrush fault current under out of synchronism, as short-circuit current is calculated with asymmetry factor of 1.8. The magneto-motive force (MMF) acting on the transformer winding is calculated at least 3.24 times because it is proportional to the square of the fault current as shown in equation (1).

$$
I_{S C}=\frac{1.8 \sqrt{2}(M V A)(10)^{2}}{\sqrt{3}(k V)\left(\% Z_{T+Z}\right)}
$$

where

First Peak of the short-circuit current (kA)

(MVA) Rated Capacity of the Transformer

$(k V)$ Line Terminal Voltage

$\% Z_{T+Z}$ Impedance of the Transformer and Grid

The three-phase transformer may be likely to be damaged by the significant electro-magnetic forces accompanied by an overheating to exceed the bearing capacity of the transformer [3].

\section{Asynchronous Simulation}

\subsection{Simulation Condition}

It is assumed that a $612 \mathrm{MW}$ synchronous generator with a direct fed exciter system and tandem compound steam turbine with a steam governor are connected to an infinite bus. The initial minimum power output of the synchronous generator is about $3 \mathrm{MW}$ and the speed of the steam turbine remains constantly.

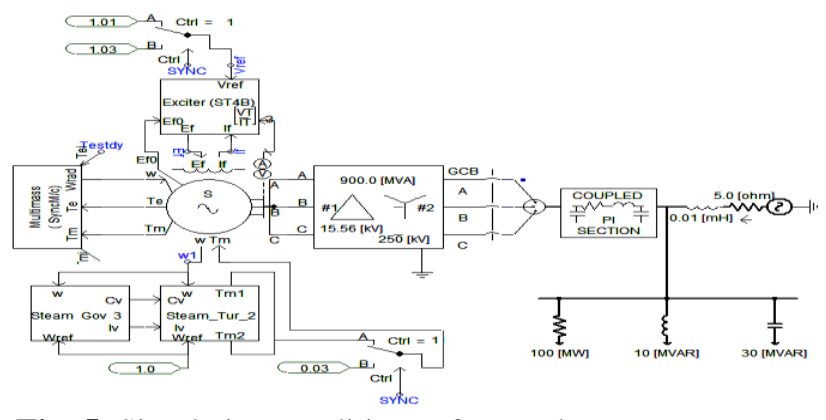

Fig. 5. Simulation conditions of a synchronous generator

A study on the dynamic behaviors of the synchronous generator was carried out using the PSCAD V4.2 software. The following Fig. 6 shows the results of the dynamic characteristics such as the terminal voltage, field current, active power and reactive power just after the synchronous generator was physically connected to an external power network.

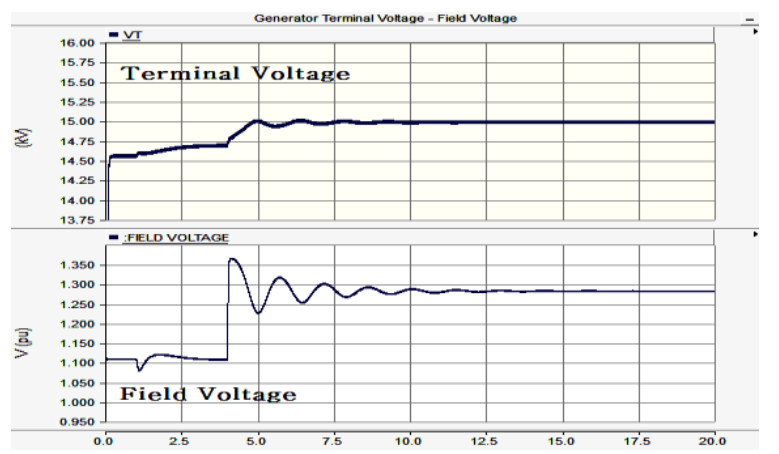

a) Terminal voltage of generator and field voltage of exciter

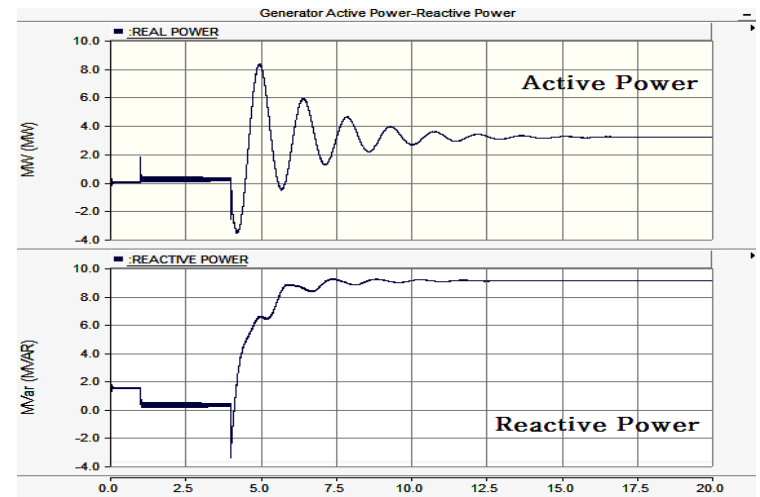

b) Active and reactive power of the synchronous generator

Fig. 6. The dynamic characteristics of the synchronous generator during normal synchronizing 
The first peak current value of the synchronous generator synchronized with the grid system is about $0.07 \mathrm{kA}$.

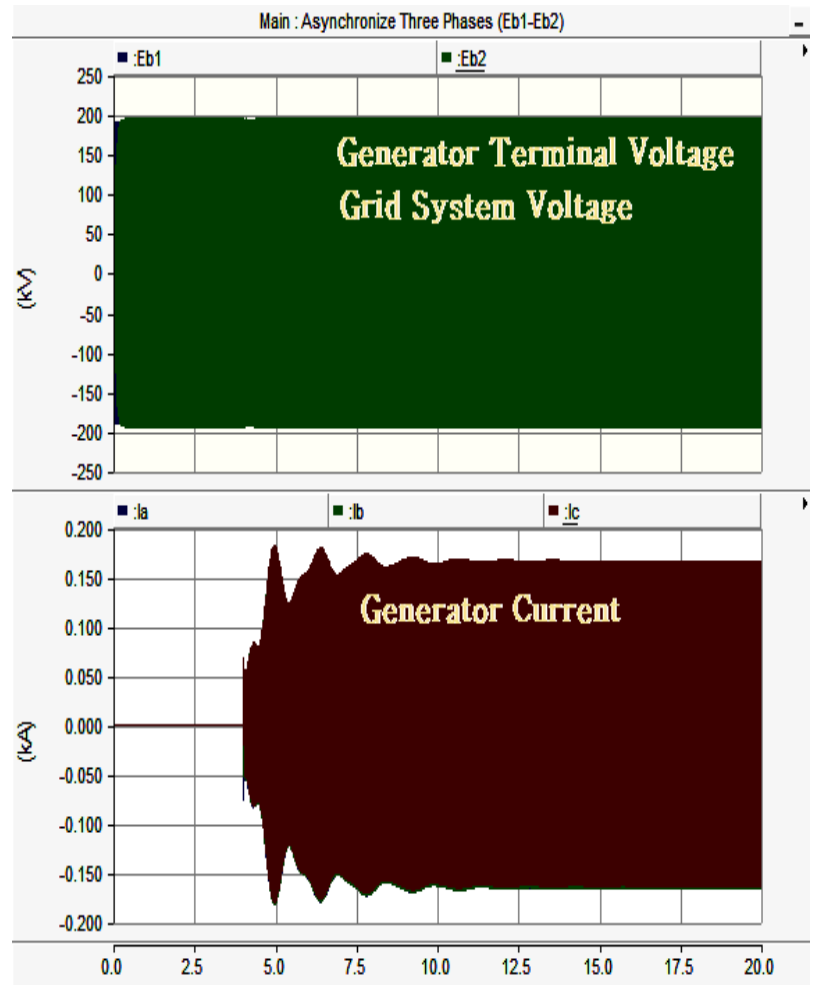

a) Voltage and current during normal synchronizing

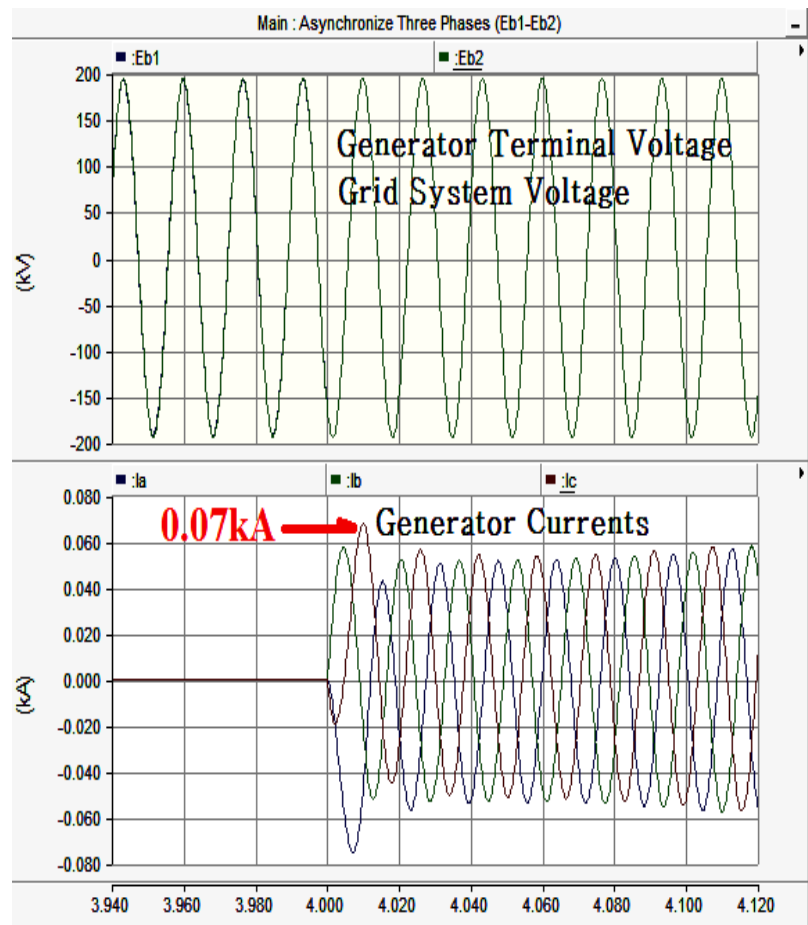

b) Peak value of voltage and current during normal syn.

Fig. 7. The peak values of voltage and current of the synchronous generator during normal synchronizing

\subsection{The phenomenon of the asynchronous}

In case the deviation of the voltage phase on both sides of the generator circuit breaker is 120 degrees, the magnitude of the transient inrush current is greater about 200 times than in synchronism condition. The fluctuation of the terminal voltage, current, and active power of the synchronous generator will be repeated periodically over a period of time as shown in the following Fig. 8-9.

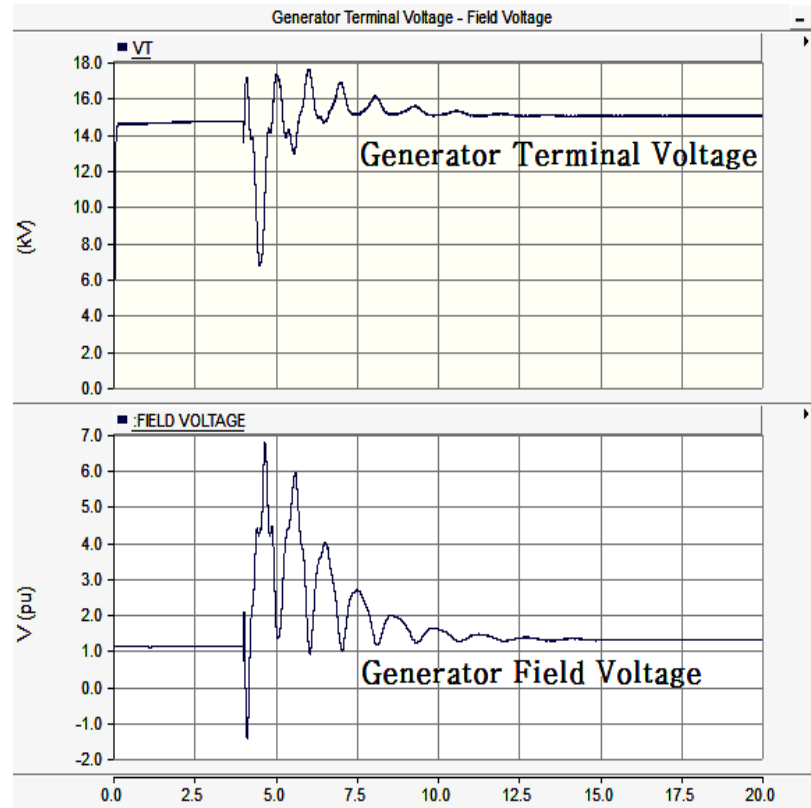

a) Transient voltage and current (120 degrees)

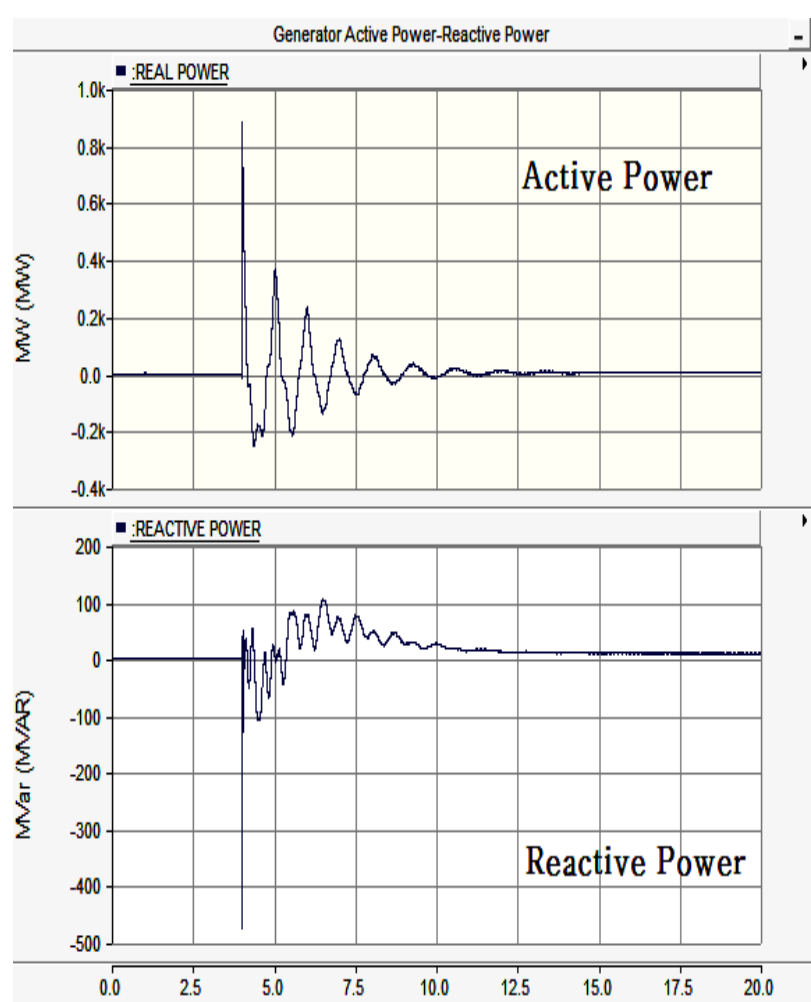

b) Transient active and reactive power (120 degrees)

Fig. 8. Transients wave form at asynchronous conditions 


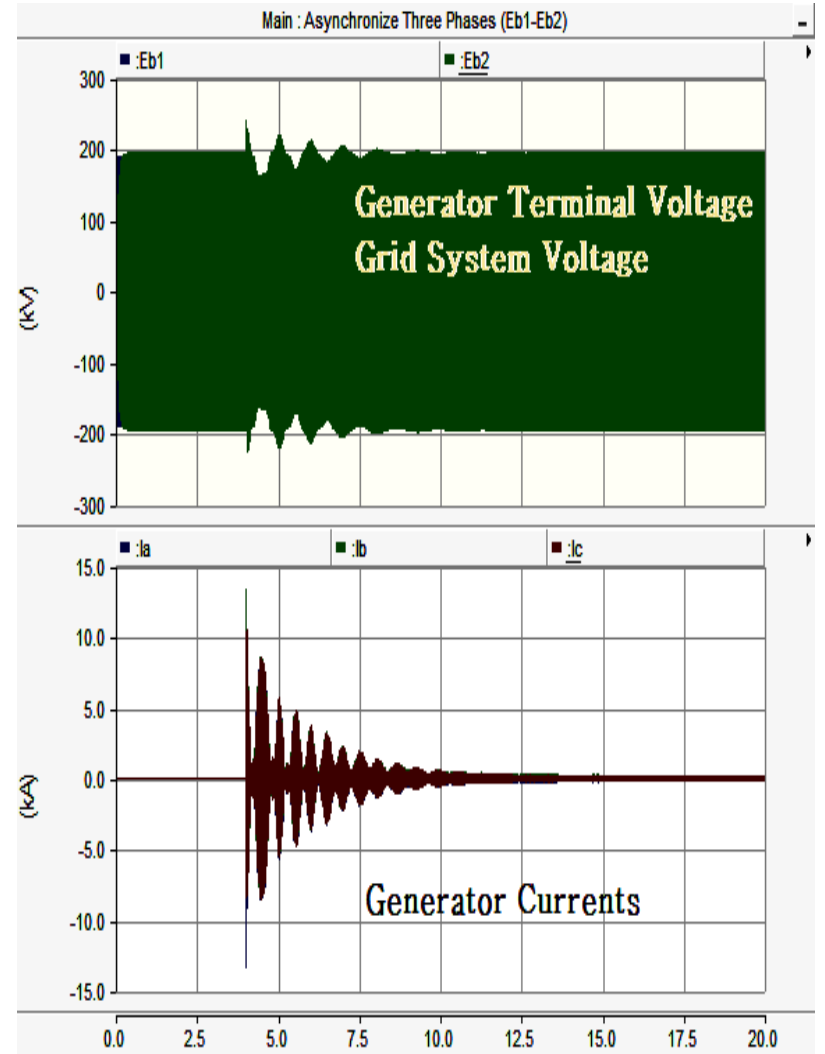

a) Transient voltage and current (120 degrees)

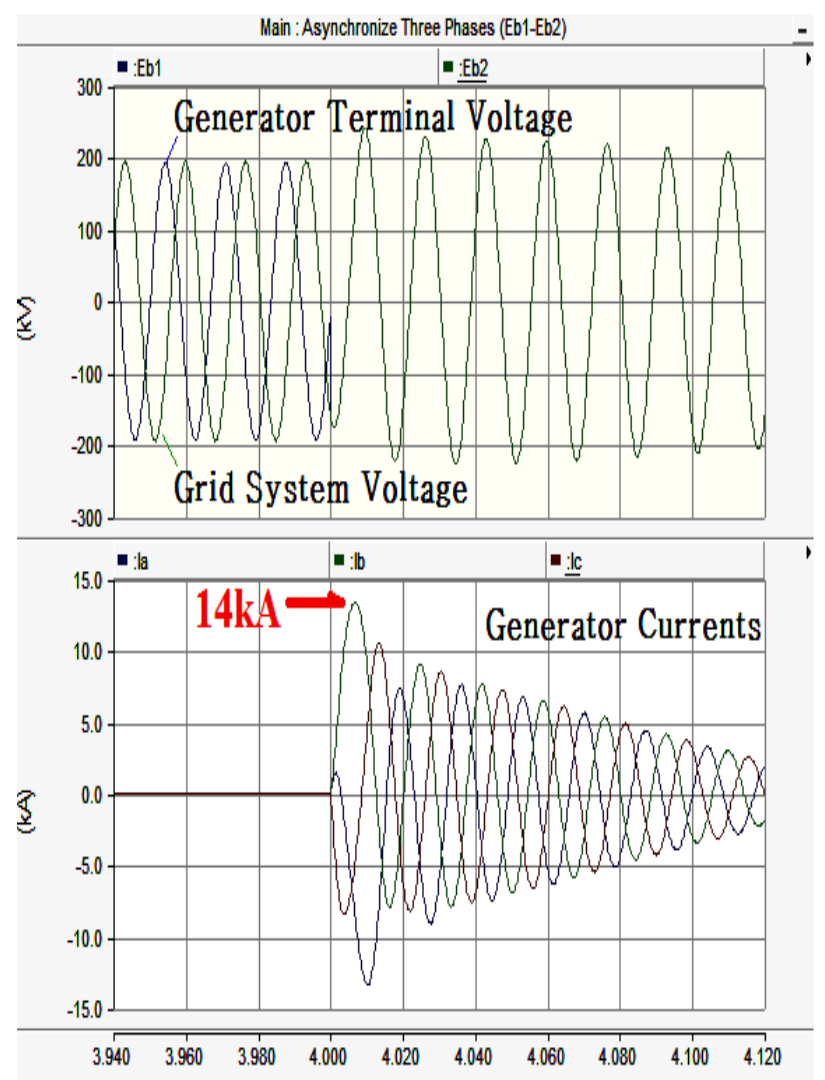

b) Peak value of transient voltage and current (120 degrees)

Fig. 9. Fluctuations of voltage and current at asynchronous conditions

\section{Asynchronous Protection}

\subsection{New asynchronous protection concepts}

It is necessary to detect the asynchronous state within a short time so as to prevent damage of the transformer from the inrush fault current. In general, the loss of excitation or the out of step protection is typically used for synchronous generators [4]. As experienced, although the loss of excitation protection took $450 \mathrm{~ms}$ until the three-phase generator circuit breakers were opened, but the windings of three-phase step-up transformer were damaged by an inrush fault current. So, the generator circuit breakers should be operated as soon as possible.

The $\alpha \beta$ transformation also known as the Clarke transformation is a mathematical transformation employed to simplify the analysis of three-phase circuits. Instantaneous values of phase voltages and line currents referred to the $a b c$ stationary axes are transformed into the $\alpha \beta$ stationary axes, or vice-versa. The $\mathrm{a}, \mathrm{b}$ and $c$ axes are spatially shifted by $2 \pi / 3 \mathrm{rad}$ from each other while the $\alpha$ and $\beta$ axes are orthogonal, and the $\alpha$ axes is parallel to the $a$ axis [5]-[6].

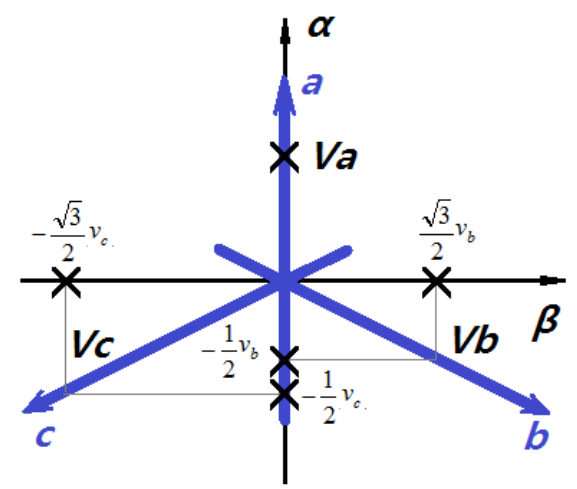

Fig. 10. The $a b c$ to $\alpha \beta$ transformation concept (Clarke transformation)

The instantaneous voltage and current vector are defined from the instantaneous $\alpha$ - and $\beta$-voltage components as shown in equation (2)-(4). Instantaneous current is obtained similarly.

$$
\begin{aligned}
& {\left[\begin{array}{l}
v_{\alpha} \\
v_{\beta}
\end{array}\right]=\sqrt{\frac{2}{3}}\left[\begin{array}{ccc}
1 & -\frac{1}{2} & -\frac{1}{2} \\
0 & \frac{\sqrt{3}}{2} & -\frac{\sqrt{3}}{2}
\end{array}\right]\left[\begin{array}{l}
v_{a} \\
v_{b} \\
v_{c}
\end{array}\right]} \\
& e=v_{\alpha}+j v_{\beta}=\sqrt{3} V e^{j\left(\omega t+\phi_{v}\right)}
\end{aligned}
$$




$$
i=i_{\alpha}+j i_{\beta}=\sqrt{3} I e^{j\left(\omega t+\phi_{I}\right)}
$$

The $a$ axis must be coincident with the $\alpha$ axis that is the real axis of the complex plane. The $\beta$ axis is the imaginary axis shifted by $\pi / 2$ from the real $\alpha$ axis. The magnitude and phase of the instantaneous voltage and current signals can be calculated. So, the admittance can be obtained easily by dividing the current into the voltage.

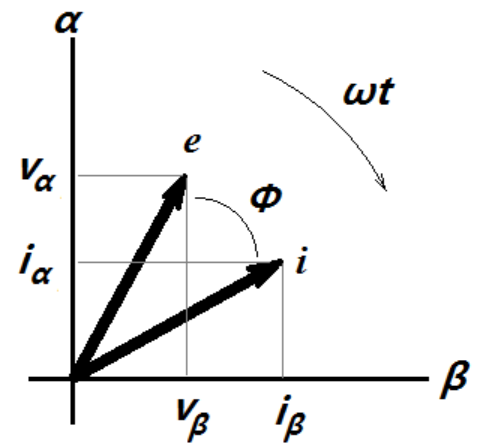

Fig. 11. Vectors of voltage and current on the $\alpha \beta$ twophase stationary reference frames

The new protection scheme for early detecting an asynchronous condition has been proposed hear based on the magnitude of the admittance signal as described in Fig. 12 below.

The asynchronous condition can be detected quickly by a surge admittance signal calculated from the measurements of the three-phase voltages and currents of the synchronous generator.
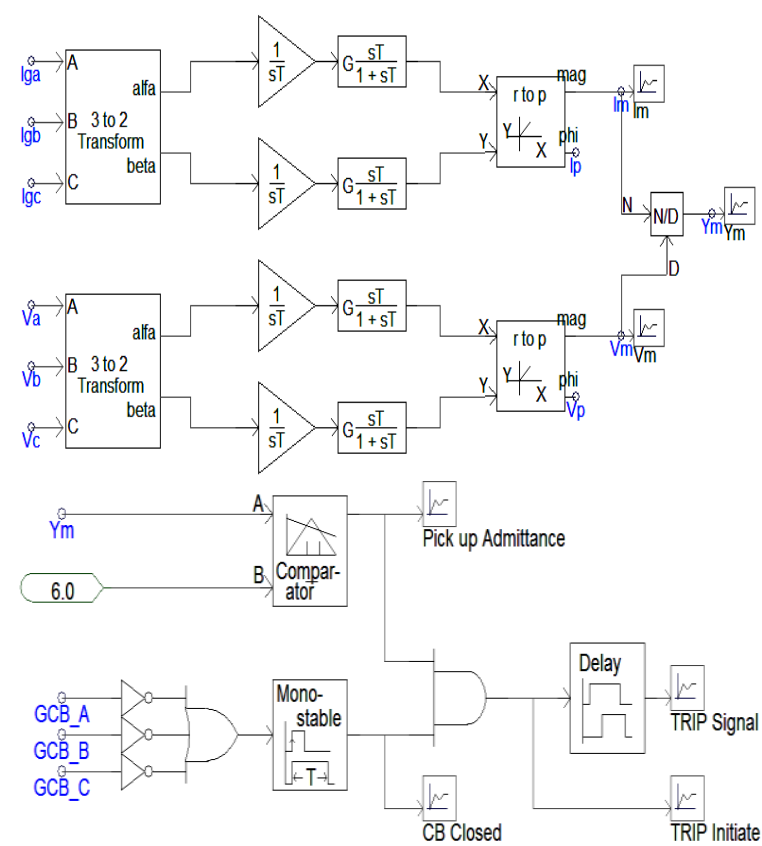

Fig. 12. The circuit of the asynchronous detection
A number of harmonics in measured signals exist in less than $1 \mathrm{kHz}$ as shown in Fig. 13. These harmonics as noise signals may lead to incorrect calculation of the asynchronous detection system.

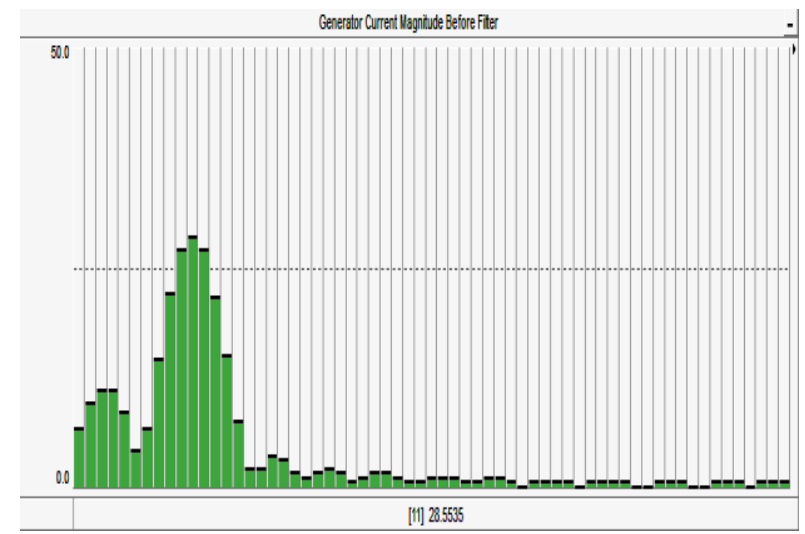

Fig. 13. Harmonics in the measured signals

So, it is necessary to remove the noise signals with a direct current component and higher order harmonics in the detecting signals. These can be eliminated by using the washout filters in this proposed circuit as shown in the Fig. 14.

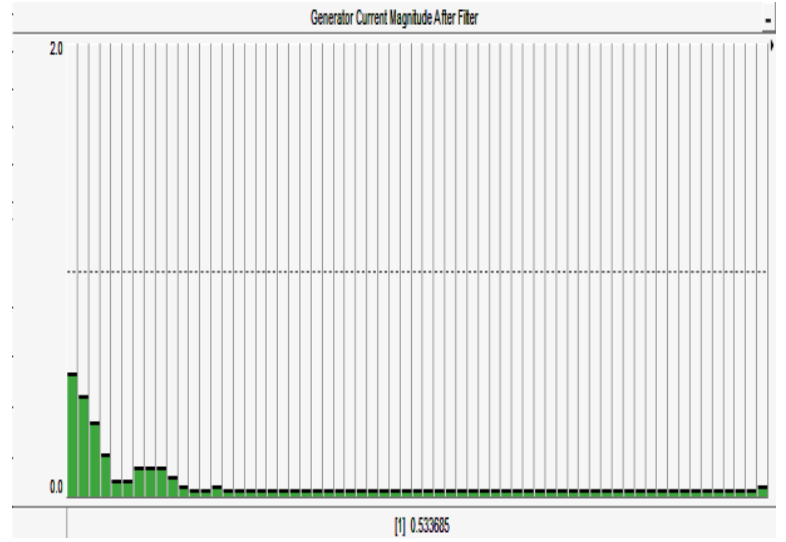

Fig. 14. The effect of the washout filter

The signal to open the generator circuit breaker is made of a combination of the admittance signal and the closed signal of the generator circuit breaker. Trip signal will be maintained for about $100 \mathrm{~ms}$ using an off-delay timer in order to use only when the synchronous generator is connected to the grid system.

\subsection{Performance of the asynchronous detection device}

When the synchronous generator is about to connect to the grid system under synchronous conditions, the trip signals of the asynchronous detection device does not occur because the magnitude of the admittance is less than the set value as shown in Fig. 15. 


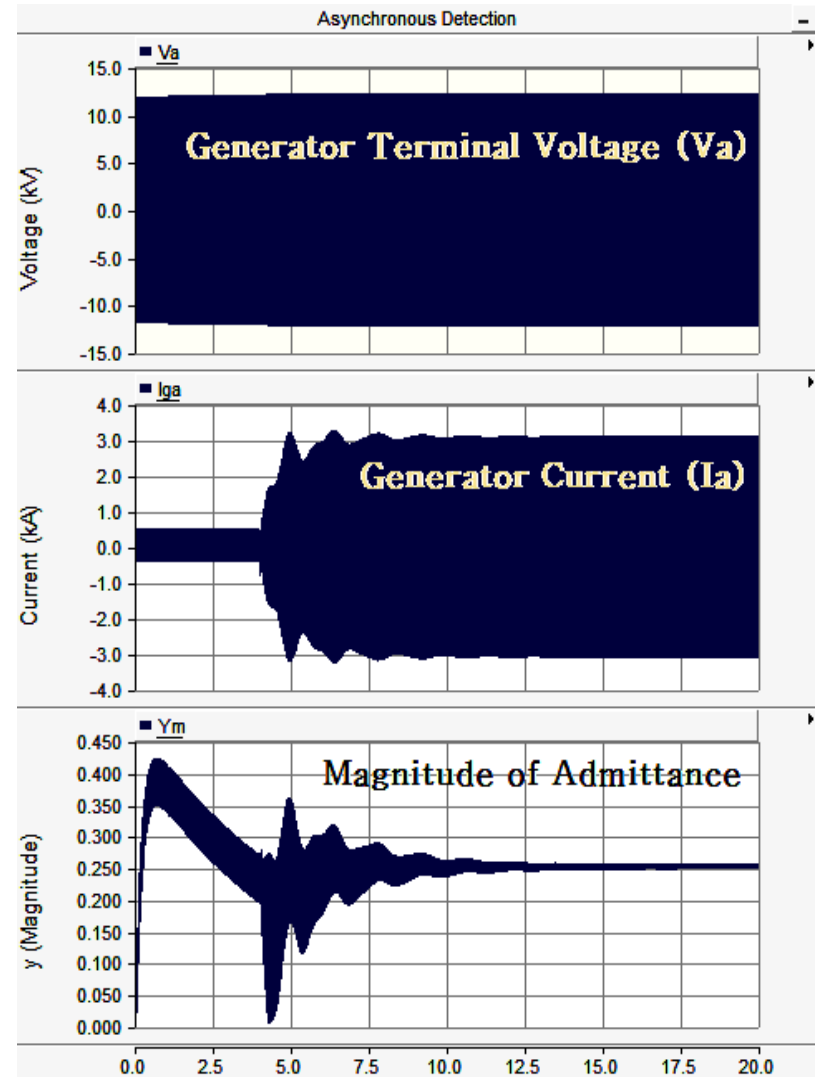

a) Voltage, current and surge admittance during normal synchronizing

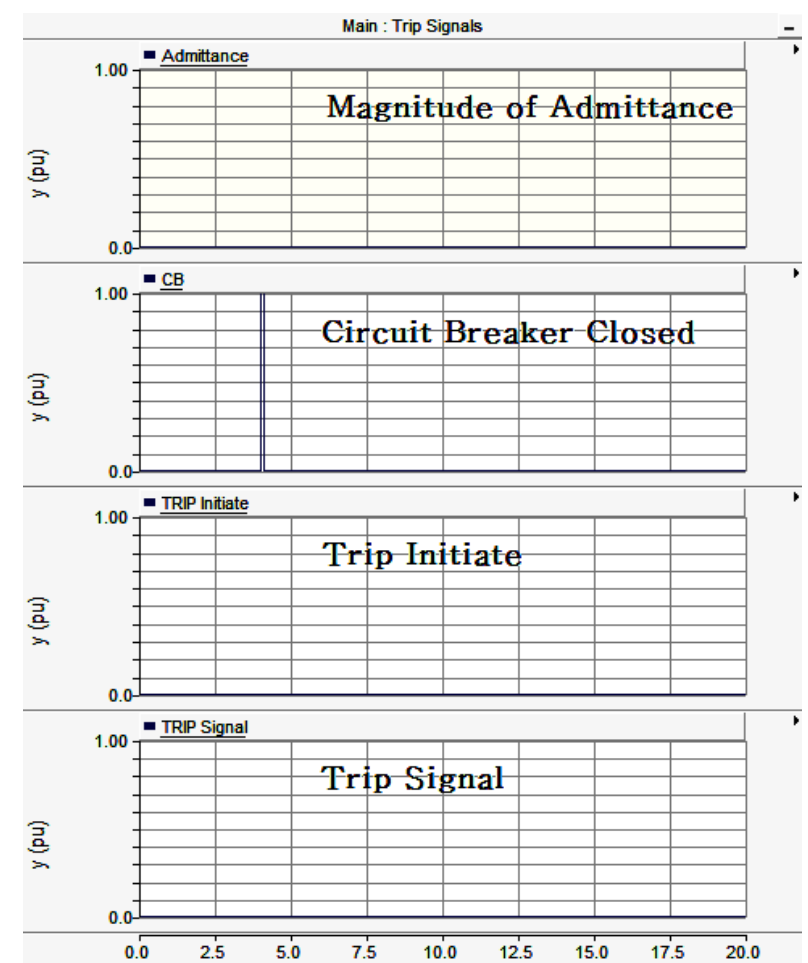

b) Signals of asynchronous detection system during normal synchronizing

Fig. 15. Dynamic behavior of the asynchronous detecting device in synchronism
If the voltage phase deviation is 120 degree between the synchronous generator and the grid system, the first cycle magnitude of the admittance signal rises rapidly up to $65 \mathrm{pu}$. Therefore, the asynchronous conditions can be early detected within short time $3.3 \mathrm{~ms}$ as shown in Fig. 16.

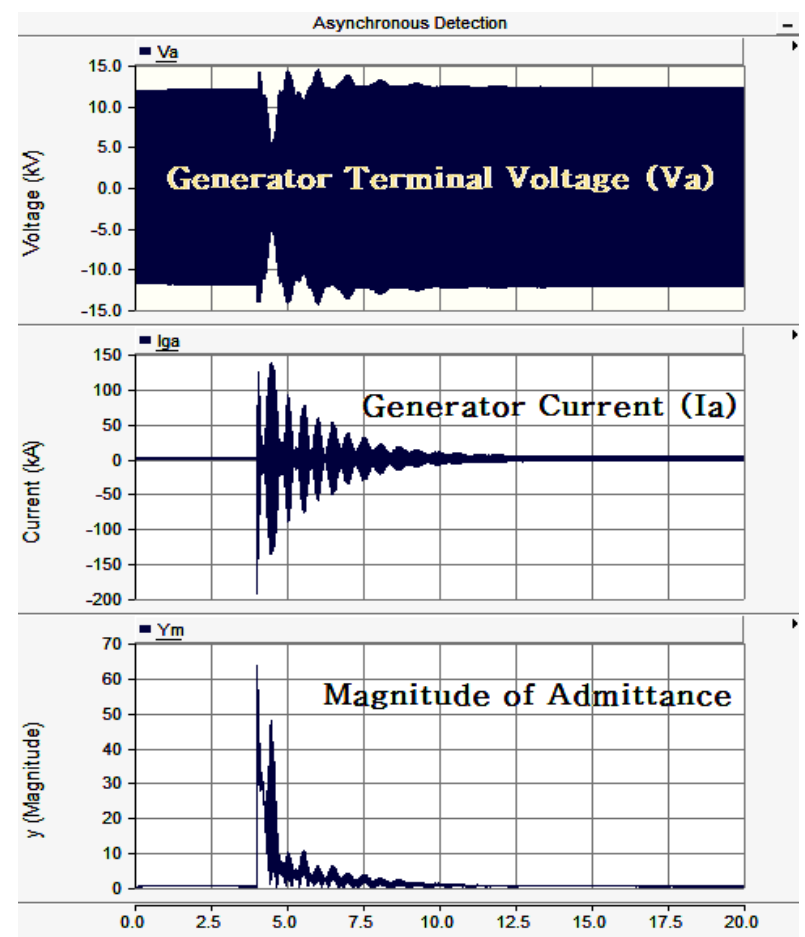

a) Voltage, current and surge admittance (120 degrees)

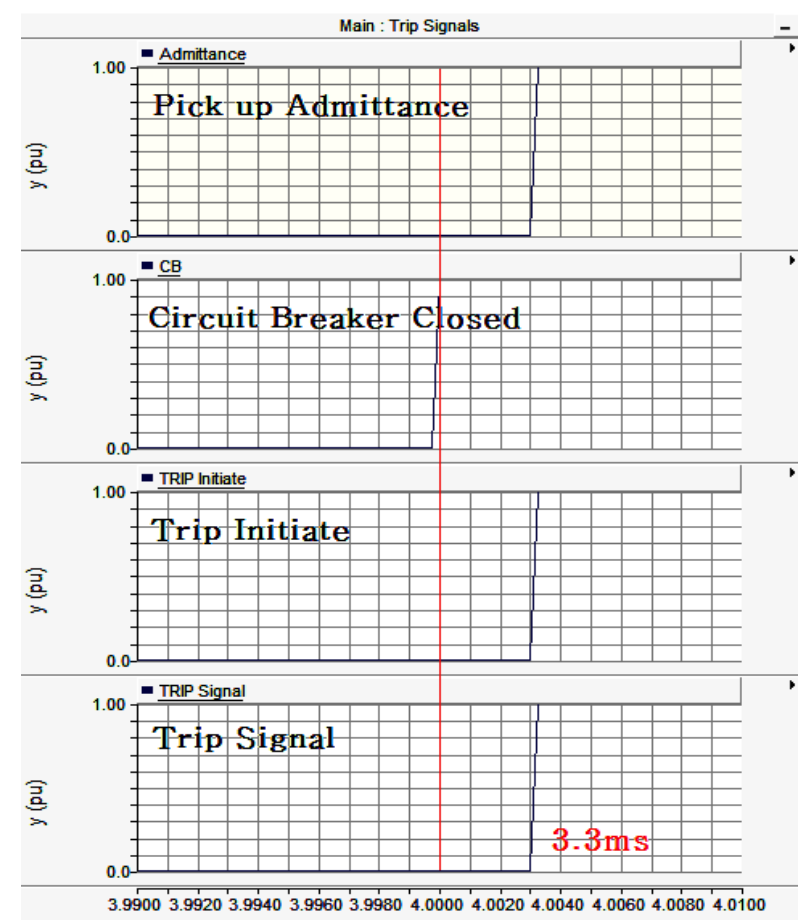

b) Signals of asynchronous conditions (120 degrees)

Fig. 16. Dynamic behavior of the asynchronous detecting device at 120 degrees 
If the voltage phase deviation is 30 degrees, the magnitude of the admittance signal is increased up to $16 \mathrm{pu}$ within $5.7 \mathrm{~ms}$.

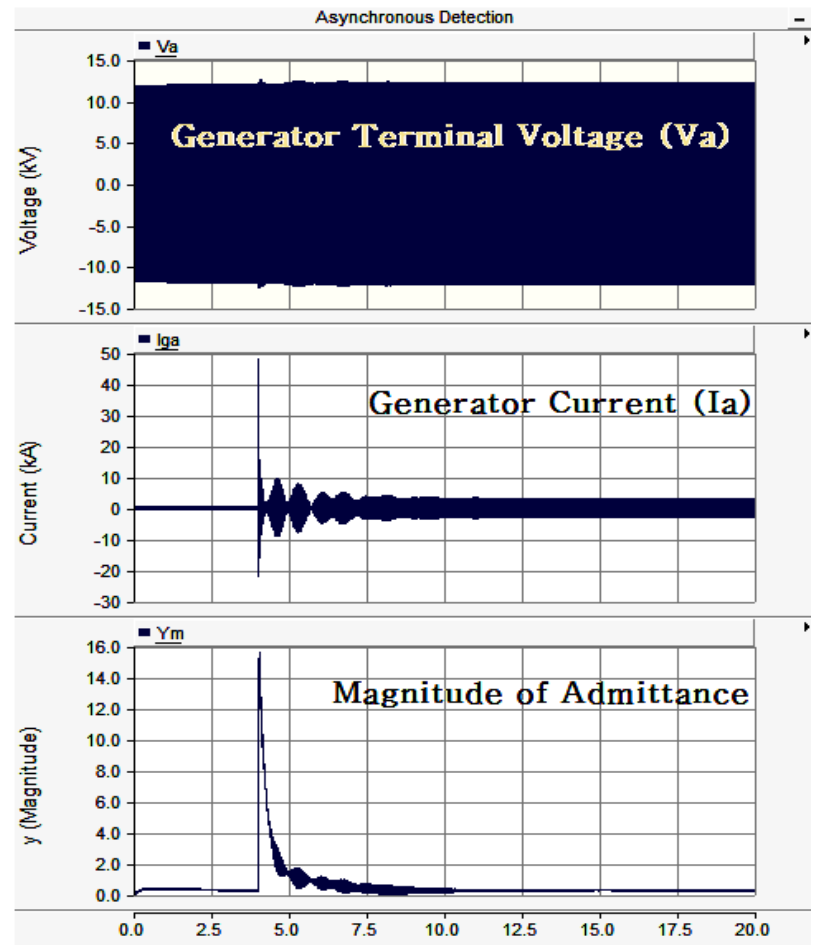

a) Voltage, current and surge admittance (30 degrees)

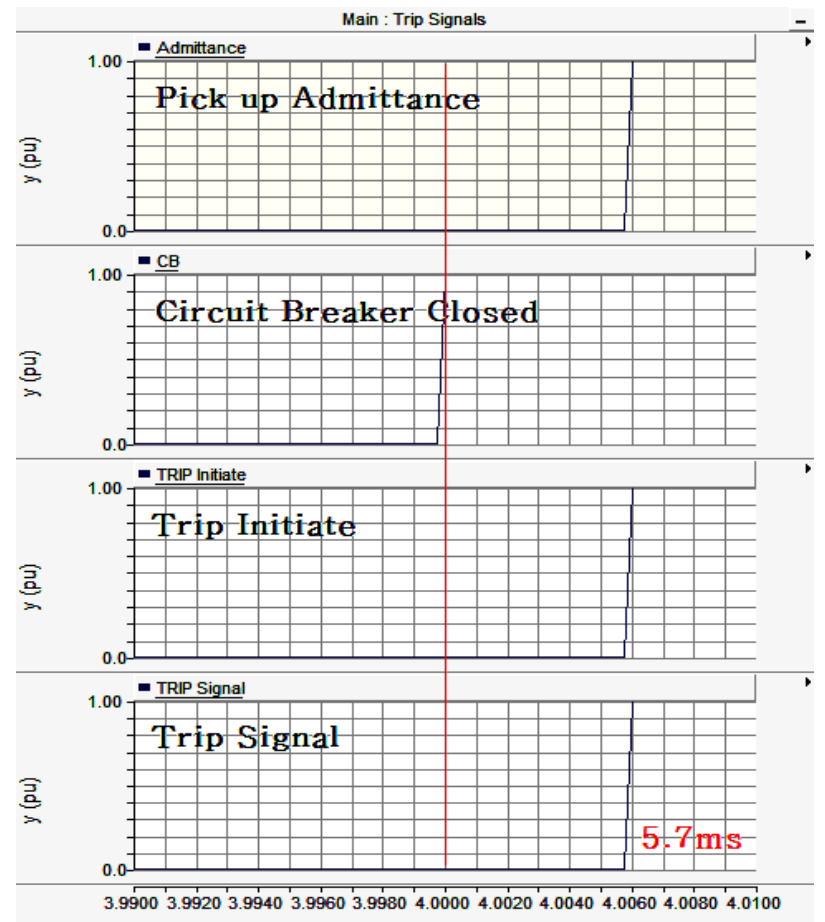

b) Signals of asynchronous conditions (30 degrees)

Fig. 17. Dynamic behavior of the asynchronous detecting device at 30 degrees
The performance of the asynchronous detection device has features to early detect asynchronous conditions. The surge admittance that has the dynamic behaviors of voltage and current is linearly proportional to the deviation of the phase angles while its operating time is exponentially decrease with inverse time characteristics as shown in Fig. 18 .

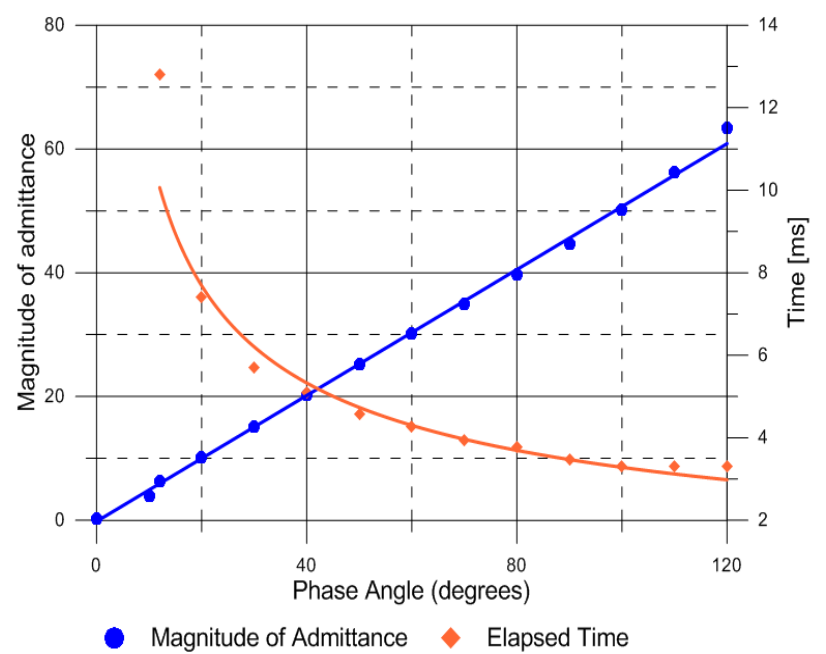

Fig 18. Surge admittance and operation time characteristics curves of the Asynchronous Detection Device.

\section{Conclusion}

The research for early detection of asynchronous state has been conducted since experiencing a severe failure of the high voltage power transformer. This proposed new protection scheme is based on the surge admittance that has a dynamic behavior of the voltage and current during the transitional period. Its performance such as early detecting asynchronous state, operating time, combination with signals of circuit breaker as well as noise reduction to ensure reliable operation has been identified as results of various simulation tests.

All components of the proposed protection devices can be implemented with only simple logic circuits or numerical calculations in a micro-processor. This new protection concept is useful to prevent the damages of the main step-up transformer from serious inrush fault currents caused by misconnection or malfunction of the synchronizing devices and human errors just after synchronous generator connected to the grid. Therefore, it can complement the blind spot of existing protections so that no equipment is damaged caused by unforeseen asynchronous problems. 


\section{References}

[1] Okamoto, "Why cannot asynchronous operation", Mitsubishi Transactions September 1986, Pp 109-114.

[2] B. R. Hill, "Modern Power Station Practice, Third Edition in 12 volumes, Electrical System and Equipment Volume D" PERGAMON PRESS, pp. 948-985

[3] A. C. Franklin, D. P. Franklin, "The J\&P Transformer Book, 11th Edition, A Practical Technology of the power transformer", Butterworths, pp. 666-680.

[4] J. A. Imhof, "Out of Step Relaying for Generators Working Group Report", IEEE Transactions on Power Apparatus and Systems, Vol. PAS-96, No.5, pp. 1556-1564, September/October 1977.

[5] E Clarke, "Circuit Analysis of A-C Power Systems", Vol. ISymmetrical and Related Components, Wiley, 1943.

[6] Hirofumi Akagi, Edson Hirokazu Watanabe, Mauricio Aredes, "Instantaneous Power Theory and Applications to Power Conditioning", WILEY-INTERSCIENCE, pp. 41-47.

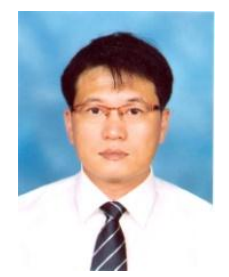

Hyung-Joo Choi received M.S. degree in electrical engineering from the ChungNam national university and $\mathrm{He}$ has worked as a manager in charge of expert service team of the power utility company, KOMIPO in Korea. His research interests are optimization and stability of power systems and electric machines.

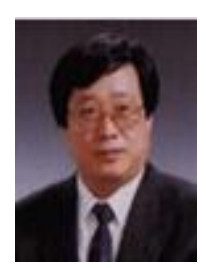

Heung-Ho Lee received Ph.D. degree in Electrical Engineering from Seoul National University, Korea and has served as a professor in the Chung-Nam National University. His research interests are computer science for power system and electric machines. 